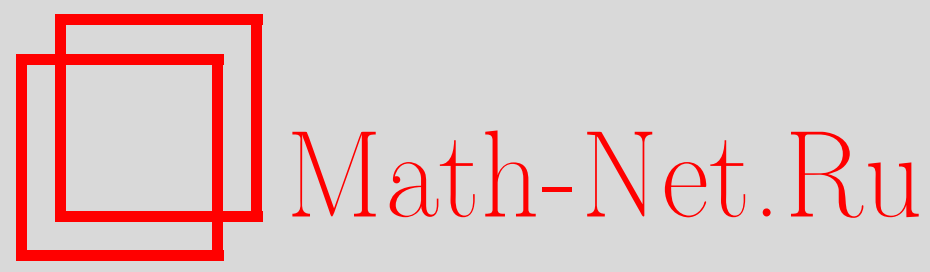

П. В. Семенов, Локальная паравыпуклость и локальная селекционная теорема, Матем. заметки, 1999, том 65, выпуск 2, 261-269

DOI: https://doi.org/10.4213/mzm1048

Использование Общероссийского математического портала Math-Net.Ru подразумевает, что вы прочитали и согласны с пользовательским соглашением http://www . mathnet.ru/rus/agreement

Параметры загрузки:

IP : 54.237 .59 .107

26 апреля 2023 г., 15:59:47

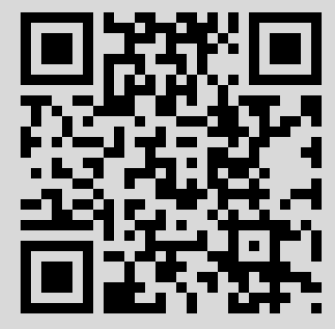




\title{
ЛОКАЛЬНАЯ ПАРАВЫПУКЛОСТЬ И ЛОКАЛЬНАЯ СЕЛЕКЦИОННАЯ ТЕОРЕМА
}

\author{
П. В. Семёнов
}

\begin{abstract}
Доказаны теоремы о возможности локального продолжения селекций для невьпуклозначных отображений паракомпактов в банаховы пространства, т.е. доказаны бесконечномерные аналоги конечномерной селекционной теоремы Майкла. Результат удается получить при надлежащем метрическом контроле за локальной степенью невыпуклости значений $F(x)$, что естественно приводит к выделению понятия равностепенно локально паравыпуклого семейства множеств. Показано, что связные подмножества интегральных кривых дифференциального уравнения $y^{\prime}=f(x, y)$ с непрерьвной правой частью $f$ вместе со своими изометрическими образами образуют равностепенно локально паравьпуклое семейство подмножеств евклидова пространства.

Библиографияя: 10 названий.
\end{abstract}

Непрерывное однозначное отображение $f: X \rightarrow Y$ называется селекиией многозначного отображсения $F: X \rightarrow Y$, если $f(x) \in F(x)$ для всех $x \in X$. Для $(n+1)$-мерного (в смысле $\operatorname{dim}$ ) паракомпакта $X$ необходимым условием существования селекций у полунепрерьвного снизу отображения $F: X \rightarrow Y$ является $n$-связность и локальная $n$-связность значений $F(x), x \in X$. Добавление “коллективного" варианта локальной связности, так называемой равностепенной локальной связности, уже дает достаточные условия существования непрерьвных селекций [1]. Тем самым, для конечномерных паракомпактов селекции существуют только при наличии соответствующих ограничений на порядок связности значений полунепрерьвного снизу многозначного отображения: исключением является только случай нульмерных областей определения. Для бесконечномерных областей определения ситуация более жесткая: недостаточно [2] потребовать стягиваемости значений $F(x)$ и равностепенной локальной стягиваемости семейства $\{F(x)\}$ значений. Более того, чисто топологические ограничения, гарантирующие наличие селекций, скорее всего отсутствуют в принципе. По крайней мере это так, если в качестве эталона иметь конечномерную селекционную теорему [3]. Итак, для многозначных отображений бесконечномерных пространств приходится использовать уже дополнительные, не топологические, ограничения. Своего рода палочкой-выручалочкой является здесь выпуклость значений многозначного отображения. Имеется ввиду либо стандартная выпуклость [4] в банаховом пространстве $Y$, либо различные аксиоматически заданные вьпуклости в метрических или топологических пространствах $Y$.

Работа выполнена при финансовой поддержке Российского фонда фундаментальных исследований, грант № 96-01-01166. 
В настоящей работе доказаны теоремы о возможности локального продолжения селекций для невыпуклозначных отображений бесконечномерных паракомпактов, т.е. доказаны бесконечномерные аналоги конечномерной локальной селекционной теоремы [1]. Результат удается получить при надлежащем метрическом контроле за локальной степенью невьпуклости значений $F(x)$. Селекционная теорема при глобальном метрическом контроле за невьпуклостью значений $F(x)$ известна [5]. По существу, в данной работе исследуются подходящие локальные варианты понятия паравыnуклости [5].

1. Для подмножества $A$ банахова пространства $Y$ будем обозначать через $[A]$ выпуклую оболочку множества $A$, а через $R(A)$ - чебышевский радиус множества $A$, т.е. инфимум множества радиусов открытых в $Y$ шаров, содержащих $A$. Для двух подмножеств $A$ и $B$ метрического пространства обозначим через $\operatorname{dist}(A: B)$ уклонение множества $A$ от множества $B$, т.е.

$$
\operatorname{dist}(A: B)=\sup _{a \in A} \inf _{b \in B}\{d(a, b)\} .
$$

Ясно, что минимум из уклонений $\operatorname{dist}(A: B)$ и $\operatorname{dist}(B: A)$ является хаусдорфовым расстоянием между множествами $A$ и $B$, порожденным заданной метрикой $d$.

ОПРЕДЕЛЕНИЕ 1. Пусть $\alpha \geqslant 0$. Будем говорить, что открытый шар $D$ банахова пространства $Y \alpha$-пересекает подмножество $L \subset Y$, если $D \cap Y \neq \varnothing$ и если $\operatorname{dist}([A]: L) \leqslant \alpha R(A)$ для всех подмножеств $A$ пересечения $D \cap L$. В случае, когда шар $D \alpha$-пересекает все элементы семейства $\{L\}$, с которыми он пересекается, будем говорить, что $D \alpha$-пересекает семейство $\{L\}$ подмножеств банахова пространства $Y$.

Отметим, что свойство шара $D \alpha$-пересекать данное семейство $\{L\}$ наследственно в том смысле, что если шар $D^{\prime}$ лежит в $D$, то и $D^{\prime} \alpha$-пересекает семейство $\{L\}$. Телом $\bigcup\{L\}$ семейства $\{L\}$ подмножеств данного множества назовем объединение всех членов этого семейства. Множество назьвается паравыпуклым [5], если для некоторого $\alpha \in[0,1)$ любой шар $D$ или не пересекает это множество, или же $\alpha$-пересекает его.

ОпредЕЛЕниЕ 2. Семейство $\{L\}$ непустых замкнутых подмножеств банахова пространства назьвается равностепенно локально паравыпуклым, если для любой точки $y$ из тела семейства $\{L\}$ найдутся $r=r(y)$ и $\alpha=\alpha(y) \in[0,1)$ такие, что шар $D(y, r)$ радиуса $r$ с центром в $y \alpha$-пересекает семейство $\{L\}$.

ТЕОрема 1. Пусть $F$ - полунепрерывное снизу отображсение паракомпакта $X$ в равностепенно локально паравыпуклое семейство $\{L\}$ замкнутых подмножеств банахова пространства $Y$. Пусть $A$ замкнуто в $Y$ и $h$ является непрерывной однозначной селекиией отображения $\left.F\right|_{A}$. Тогда найдется открытое множество $U \supset A$ и непрерывная однозначная селекиия $f$ отображения $\left.F\right|_{U}$, являющаяся продолжением частичной селекчии $h$.

Естественна версия теоремы 1, в которой невыпуклость оценивается в точках из множества значений $h(A)$ частичной селекции $h$ отображения $F$.

ОПРЕДЕЛЕНИЕ 3. Для семейства $\{L\}$ непустых замкнутых подмножеств банахова пространства и любой точки $y \in \bigcup\{L\}$ локальной невыпуклостью семейства $\{L\}$ в точке $y$ назьвается число

$$
\alpha_{\text {loc }}(y,\{L\})=\varlimsup_{r \rightarrow 0} \inf \{\alpha \geqslant 0\},
$$

где инфимум берется по всем шарам $D(y, r), \alpha$-пересекаюшим $\{L\}$. 
ТЕОрема 2. Теорема 1 верна при замене условия равностепенной локальной паравыпуклости семейства $\{L\}$ на условие $\alpha_{\mathrm{loc}}(h(a),\{L\})<1$ для всех $a \in A$.

В случае одного непустого замкнутого подмножества $L$ банахова пространства определение 3 приводит к понятию локальной невьпуклости $\alpha_{\operatorname{loc}}(y, L)$ множества $L$ в точке $y \in L$.

В п. 2 доказана теорема 1 , а в п. 3 собраны доказательства используемых в п. 2 вспомогательных утверждений и доказана теорема 2. В п. 4 приведены примеры локально паравьпуклых множеств и равностепенно локально паравьпуклых семейств множеств. Наконец, напомним, что многозначное отображение $F: X \rightarrow Y$ топологических пространств назьвается полунепрерывным снизу, если в $X$ открыт прообраз $F^{-1}(U)$ любого открытого в $Y$ множества $U$; при этом (полный) прообраз $F^{-1}(U)$ множества $U$ определяется как множество всех тех $x \in X$, для которых значения $F(x)$ пересекают множество $U$.

2. ДОКАЗАТЕЛЬСТво ТЕОРЕМЫ 1. Пусть $\{L\}$ - равностепенно локально паравыпуклое семейство подмножеств банахова пространства $Y$. Для всех чисел $r>0$ и $\alpha \in$ $[0,1)$ определим следующее подмножество $G(r, \alpha)$ тела семейства $\{L\}$. Точка $y$ из тела семейства $\{L\}$, по определению, принадлежит множеству $G(r, \alpha)$, если для некоторых $r^{\prime}>r$ и $\alpha^{\prime} \leqslant \alpha$ открытый шар $D\left(y, r^{\prime}\right)$ радиуса $r^{\prime}$ с центром в точке $y \alpha^{\prime}$-пересекает семейство $\{L\}$.

ЛЕмма 1. Справедливы следующие утверждения:

а) если $R \leqslant r \quad$ u $\beta \geqslant \alpha, \operatorname{mo} G(R, \beta) \supset G(r, \alpha)$;

б) $\bigcup\{L\}=\bigcup\{G(r, \alpha) \mid r>0,0 \leqslant \alpha<1\}$;

в) множества $G(r, \alpha)$ открыты в $\cup\{L\}$.

Доказательство теоремы разобьем на два случая. В первом "равномерном" случае мы предположим, что тело $\bigcup\{L\}$ семейства $\{L\}$ совпадает с некоторым из множеств $G(r, \alpha)$. Во втором случае мы представим тело $\bigcup\{L\}$ семейства $\{L\}$ в виде растущей последовательности открытых множеств $G_{n}=G\left(r_{n}, \alpha_{n}\right)$ для некоторой монотонно убывающей к 0 последовательности $\left\{r_{n}\right\}$ положительных чисел и некоторой монотонно возрастающей к 1 последовательности $\left\{\alpha_{n}\right\}$ чисел из промежутка $[0,1)$.

1) $\cup\{L\}=G(r, \alpha)$ для некоторых $r>0$ и $\alpha \in[0,1)$. Фиксируем $\beta \in(\alpha, 1)$ и положим

$$
\varepsilon=r \frac{2(1-\beta)}{3-\beta}>0 .
$$

Произвольно продолжим $h: A \rightarrow Y$ до непрерывного отображения (необязательно селекции) $h^{*}: X \rightarrow Y$. Для каждой точки $a \in A$ определим ее окрестность:

$$
V(a)=F^{-1}\left(D\left(h(a), \frac{\varepsilon}{2}\right)\right) \cap\left(h^{*}\right)^{-1}\left(D\left(h(a), \frac{\varepsilon}{2}\right)\right) \subset X .
$$

Пусть $U$ - окрестность множества $A$, замыкание которой лежит в объединении всех окрестностей $V(a)$. Тогда ограничение отображения $h^{*}$ на замькание окрестности $U$ есть непрерьвное однозначное отображение с паракомпактной областью определения, являющееся $\varepsilon$-селекцией соответствующего ограничения отображения $F$. Действительно, если $x \in V(a)$ для некоторого $a \in A$, то $\left\|h^{*}(x)-h(a)\right\|<\varepsilon / 2$ и множество $F(x)$ пересекает шар $D(h(a), \varepsilon / 2)$. Более того, $\varepsilon$-шары $D\left(h^{*}(x), \varepsilon\right) \quad \alpha$-пересекают множества $F(x), x \in \mathrm{Cl}(U)$. 
Действительно, если $x \in V(a)$ для некоторого $a \in A$, то шар $D\left(h^{*}(x), \varepsilon\right)$ лежит в шаре $D(h(a), \varepsilon+\varepsilon / 2)$, который лежит в шаре $D(h(a), r)$ в силу равенства

$$
r=\varepsilon \frac{3-\beta}{2(1-\beta)}=\frac{\varepsilon}{2}+\varepsilon\left(1+\beta+\beta^{2}+\cdots\right)>\varepsilon+\frac{\varepsilon}{2} .
$$

Согласно условию $\bigcup\{L\}=G(r, \alpha)$, т.е. для некоторых $r^{\prime}>r$ и $\alpha^{\prime} \leqslant \alpha$ открытьй шар $D\left(h(a), r^{\prime}\right) \quad \alpha^{\prime}$-пересекает семейство $\{L\}$ и, в частности, $\alpha^{\prime}$-пересекает множество $F(x)$. Так как шар $D=D\left(h^{*}(x), \varepsilon\right)$ лежит в шаре $D\left(h(a), r^{\prime}\right)$, то $D \alpha^{\prime}$-пересекает множество $F(x)$, а значит, и $\alpha$-пересекает множество $F(x)$. Отметим, что условие равностепенной локальной паравыпуклости использовалось только в точках множества $h(a) \subset \cup\{L\}$.

Искомое продолжение $f$ отображения $h$, являющееся селекцией отображения $\left.F\right|_{U}$, построим как равномерньй предел некоторой последовательности $f_{n}: \mathrm{Cl}(U) \rightarrow Y$, в которой $f_{0}=\left.h^{*}\right|_{\mathrm{Cl}(U)}$. Итак, по индукции построим отображения $f_{n}: \mathrm{Cl}(U) \rightarrow Y$ так, чтобы для всех $n \in \mathbb{N}$ и всех $x \in \mathrm{Cl}(U)$ :

$\left.\mathrm{i}_{n}\right)\left\|f_{n}-f_{n-1}\right\| \leqslant \beta^{n-1} \varepsilon ;$

$\left.\mathrm{ii}_{n}\right)$ шары $D\left(f_{n}(x), \beta^{n} \varepsilon\right)$ пересекали множества $F(x)$;

$\left.\mathrm{iii}_{n}\right)$ шары $D\left(f_{n}(x), \beta^{n} \varepsilon\right)$-пересекали множества $F(x)$.

Формально полагая $f_{-1}=f_{0}=h^{*}$, получаем, что эти условия выполнены при $n=0$. Действительно, условие $\mathrm{i}_{0}$ ) тривиально, а условия $\mathrm{ii}_{0}$ ) и $\mathrm{iii}_{0}$ ) уже проверены. Допустим, что отображения $f_{-1}, f_{0}, f_{1}, \ldots, f_{n}$ уже построены так, что выполняются условия $\left.\left.\mathrm{i}_{m}\right)-\mathrm{iii}_{m}\right)$ для $0 \leqslant m \leqslant n$. Определим многозначное отображение $F_{n+1}: \mathrm{Cl}(U) \rightarrow Y$ равенством

$$
F_{n+1}(x)=\operatorname{Cl}\left(\left[D\left(f_{n}(x), \beta^{n} \varepsilon\right) \cap F(x)\right]\right) .
$$

Значения этого отображения замкнуты, выпуклы и непусты по условию $\mathrm{ii}_{n}$ ), а само это отображение полунепрерьвно снизу в силу непрерьвности $f_{n}$ и полунепрерывности снизу $F$. Согласно классической вьпуклозначной селекционной теореме [4] отображение $F_{n+1}$ имеет селекцию $f_{n+1}: \mathrm{Cl}(U) \rightarrow Y$. Условие $\left.\mathrm{i}_{n+1}\right)$ вьполнено, так как $f_{n+1}(x) \in F_{n+1}(x) \subset \mathrm{Cl}\left(D\left(f_{n}(x), \beta^{n} \varepsilon\right)\right)$. Условие $\left.\mathrm{ii}_{n+1}\right)$ есть следствие условия iii $\left._{n+1}\right)$, поскольку

$$
\begin{aligned}
\operatorname{dist}\left(f_{n+1}(x), F(x)\right) & \leqslant \operatorname{dist}\left(\left[D\left(f_{n}(x), \beta^{n} \varepsilon\right) \cap F(x)\right]: F(x)\right) \\
& \leqslant \alpha R\left(\left[D\left(f_{n}(x), \beta^{n} \varepsilon\right) \cap F(x)\right]\right) \leqslant \alpha \beta^{n} \varepsilon<\beta^{n+1} \varepsilon .
\end{aligned}
$$

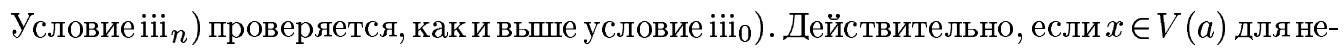
которого $a \in A$, тошар $D\left(f_{n+1}(x), \beta^{n+1} \varepsilon\right)$ лежит в шаре $D\left(h(a), \varepsilon / 2+\varepsilon\left(1+\beta+\cdots+\beta^{n}\right)\right)$, так как

$$
\left\|f_{n+1}(x)-h^{*}(x)\right\|=\left\|f_{n+1}(x)-f_{0}(x)\right\| \leqslant \sum_{m=0}^{n}\left\|f_{m+1}(x)-f_{m}(x)\right\| \leqslant \varepsilon\left(1+\beta+\cdots+\beta^{n}\right)
$$

в силу условий $\left.\left.\mathrm{i}_{0}\right)-\mathrm{i}_{n+1}\right)$.

В свою очередь, из

$$
r=\varepsilon \frac{3-\beta}{2(1-\beta)}=\frac{\varepsilon}{2}+\varepsilon\left(1+\beta+\beta^{2}+\cdots\right)
$$


следует, что шар $D\left(h(a), \varepsilon / 2+\varepsilon\left(1+\beta+\cdots+\beta^{n}\right)\right)$ лежит в шаре $D(h(a), r)$, и дальнейшее рассуждение повторяет доказательство условия $\left.\mathrm{ii}_{0}\right)$.

Условия $\left.\mathrm{i}_{n}\right), n \in \mathbb{N}$, гарантируют фундаментальность построенной функциональной последовательности и, значит, непрерьвность поточечного предела $f$ этой последовательности. Включение $f(x) \in F(x)$ следует из условий $\left.\mathrm{ii}_{n}\right), n \in \mathbb{N}$, и полноты множеств $F(x)$. Случай 1$)$ в теореме 1 доказан.

2) Не существует $r>0$ и $\alpha \in[0,1)$ таких, что $\{L\}=G(r, \alpha)$. Пусть $r_{n}=2^{-n}$ и $\alpha_{n}=1-n^{-1}, n \in \mathbb{N}$ и $G_{n}=G\left(r_{n}, \alpha_{n}\right)$. Так как семейство $\{L\}$ равностепенно локально паравыпуклое, метрическое пространство $M=\bigcup\{L\}$ есть объединение возрастающей последовательности $\left\{G_{n}\right\}$ своих собственных открытых подмножеств $G_{n}$. Построим открытые подмножества $W_{n} \subset G_{n}$ так, чтобы $\mathrm{Cl}\left(W_{n}\right) \subset G_{n}, \mathrm{Cl}\left(W_{n}\right) \subset W_{n+1}$ и $\cup W_{n}=M$. Для этого достаточно, например, рассмотреть ограниченную метрику $\rho$ на $M$, совместимую с заданной в $Y$ топологией, и положить

$$
W_{n}=\left\{x \in M \mid \operatorname{dist}_{\rho}\left(x, M \backslash G_{n}\right)>\frac{d}{n+1}\right\}, \quad d=\sup _{x \in M}\left\{\operatorname{dist}_{\rho}\left(x, M \backslash G_{1}\right)\right\}>0 .
$$

Пусть $h: A \rightarrow Y$ - данная селекция отображения $\left.F\right|_{A}$ и $E_{n}=h^{-1}\left(W_{n}\right) \subset A \subset X$. Так как $\bigcup W_{n}=M=\bigcup\{L\}$, то открытые (в $A$ ) множества $E_{n}$ можно считать непустыми, покрывающими в объединении все множество $A$, и при этом $\mathrm{Cl}_{A}\left(E_{n}\right)=A_{n} \subset E_{n+1}$. Мы индуктивно будем продолжать селекции с $A_{n}$ на некоторые окрестности $U_{n} \supset A_{n}$ так, чтобы $U_{n+1} \supset U_{n}$. Построение основано на следующем утверждении, являющемся некоторым уточнением разобранного вьше случая 1) теоремы 1.

ПРЕДЛОЖЕНИЕ 1. Пусть $\Phi: X \rightarrow Y$ - полунепрерывное снизу отображсение паракомпакта $X$ в банахово пространство $Y, A$ - замкнутое подмнохество $X$, а $C, B, C \subset B,-$ oткрытые в $A$ подмножсества $A$ mакие, что $\mathrm{Cl}_{A}(C) \subset B$; $\varphi: A \rightarrow Y$ - непрерывная селекиия ограничения $\left.\Phi\right|_{A}$. Пусть для некоторых $R>0$, $\mu \in[0,1)$ все $2 R$-иары с иентрами в точках из $\varphi\left(\mathrm{Cl}_{A}(C)\right)$ н-пересекают семейство $\{\Phi(x)\}, x \in X$, а все $R$-шары с иентрами из $\varphi(B) \quad \mu$-пересекают то жее семейство. Тогда найдутся открытое в $X$ множество $U \supset \mathrm{Cl}_{A}(C)$ и непрерывная селекиия $\psi: \mathrm{Cl}(U) \cup A \rightarrow Y$ отображения $\Phi$ такие, что

a) $\left.\psi\right|_{A}=\varphi$;

б) $R$-иары с иентрами из $\psi(\mathrm{Cl}(U))$ н-пересекают семейство $\{\Phi(x)\}$;

в) $\mathrm{Cl}(U) \cap(A \backslash B)=\varnothing$.

Вернемся к доказательству случая 2) теоремы 1. Используем предложение 1 для $\Phi=F, \varphi=h, C=E_{1} \subset \mathrm{Cl}_{A}\left(E_{1}\right) \subset E_{2}=B, 2 R=r_{1}, \mu=\alpha_{2}$. По построению $h\left(E_{i}\right) \subset W_{i} \subset G_{i}=G\left(r_{i}, \alpha_{i}\right), i=1,2$, т.е. $r_{i}$-шары с центрами в точках из $h\left(E_{i}\right)$ $\alpha_{i}$-пересекают семейство множеств $\{L\}$. Так как $\alpha_{1}<\alpha_{2}=\mu$, можно считать, что эти шары $\mu$-пересекают семейство $\{L\}$. Кроме того, $r_{2}=r_{1} / 2$, значит предложение 1 действительно применимо. Поэтому найдутся открытое в $X$ множество $U_{1} \supset \mathrm{Cl}_{A}\left(E_{1}\right)$, замыкание которого непересекается с $A \backslash E_{2}$, и непрерывная селекция $f_{1}: \operatorname{Cl}\left(U_{1}\right) \cup A \rightarrow Y$ отображения $F$, продолжающая $h$, такие, что $r_{2}$-шары с центрами из $f_{1}\left(\mathrm{Cl}\left(U_{1}\right)\right) \alpha_{2}$-пересекают семейство $\{F(x)\}$. Используем предложение 1 для $\Phi=F$, подмножества $\mathrm{Cl}\left(U_{1}\right) \cup A$ паракомпакта $X$, частичной селекции $\varphi=f_{1}: \mathrm{Cl}\left(U_{1}\right) \cup A \rightarrow Y, C=U_{1} \cup E_{2} \subset B=U_{1} \cup E_{3}$, $2 R=r_{2}, \mu=\alpha_{3}$. Применимость предложения 1 следует из свойств частичной селекции $f_{1}$ и определения множеств $E_{2}$ и $E_{3}$. Поэтому найдутся окрестность $U_{2}($ в $X)$ множества $\mathrm{Cl}\left(U_{1}\right) \cup \mathrm{Cl}_{A}\left(E_{2}\right)$, замькание которой не пересекается с $A \backslash E_{3}$, и непрерьвная 
селекция $f_{2}: \mathrm{Cl}\left(U_{2}\right) \cup A \rightarrow Y$ отображения $F$, продолжающая селекцию $f_{1}$, такие, что $r_{3}$-шары с центрами из $f_{2}\left(\mathrm{Cl}\left(U_{2}\right)\right) \alpha_{3}$-пересекают семейство $\{F(x)\}$.

Индуктивное продолжение этой процедуры дает монотонно возрастающую последовательность $\left\{U_{n}\right\}$ открытых в $X$ множеств таких, что $U_{n+1} \supset\left(\mathrm{Cl}\left(U_{n}\right) \cup \mathrm{Cl}_{A}\left(E_{n+1}\right)\right)$, и последовательность частичньх селекций $f_{n}: \mathrm{Cl}\left(U_{n}\right) \cup A \rightarrow Y$ отображения $F$ таких, что $f_{n+1}$ продолжает $f_{n}$ и каждая $f_{n}$ продолжает $h$. Так как $E_{n}=h^{-1}\left(W_{n}\right) \subset A \subset X$ и $\cup W_{n}=\cup\{L\}$, то $\cup E_{n}=A$. Поэтому объединение $U=\cup\left\{U_{n} \mid n \in \mathbb{N}\right\}$ является окрестностью множества $A$, на которую продолжается частичная селекция $h$. Теорема 1 доказана.

3. ДокАЗАТЕЛЬСтво лЕммы 1. Свойство а) есть прямое следствие определения, а свойство б) есть переформулировка равностепенной локальной паравьпуклости семейства $\{L\}$. Докажем свойство в). Для данной точки $y \in G=G(r, \alpha)$ фиксируем $r^{\prime}>r$ и $\alpha^{\prime} \leqslant \alpha$ так, чтобы открытый шар $D\left(y, r^{\prime}\right) \alpha^{\prime}$-пересекал семейство $\{L\}$. Выберем $R \in\left(r, r^{\prime}\right)$ и положим $\delta=R-r$. Тогда если $\left\|y^{\prime}-y\right\|<\delta$, то $D\left(y^{\prime}, R\right) \subset D\left(y, r^{\prime}\right)$. Поэтому если $L \cap D\left(y^{\prime}, R\right) \neq \varnothing$, то из $A \subset L \cap D\left(y^{\prime}, R\right) \subset D\left(y, r^{\prime}\right)$ следует, что $\operatorname{dist}([A]: L) \leqslant \alpha^{\prime} R(A)$. Следовательно, шар $D\left(y^{\prime}, R\right)$ с $R>r \alpha^{\prime}$-пересекает множество $L$ с $\alpha^{\prime} \leqslant \alpha$. Поэтому все точки, $\delta$-близкие к точке $y \in G$, также лежат в $G$. Лемма 1 доказана.

ДОКАЗАТЕЛЬСТВО ПРЕДЛОЖЕНИЯ 1. ПроИЗвоЛЬНО ПродоЛЖим частичную селеКцию $\varphi: A \rightarrow Y$ отображения $\Phi$ до непрерывного отображения (не селекции) $\varphi^{*}: X \rightarrow Y$ и фиксируем число $\lambda \in(\mu, 1)$. Отделим в пространстве $X$ непересекающиеся замкнутые множества $\mathrm{Cl}_{A}(C)$ и $A \backslash B$ открытыми множествами $U_{0} \supset \mathrm{Cl}_{A}(C)$ и $U_{-1} \supset A \backslash B$ соответственно. Положим, как и вьше,

$$
\varepsilon=R \frac{2(1-\lambda)}{3-\lambda}>0
$$

Для каждой точки $c \in \mathrm{Cl}_{A}(C)$ определим открытое множество

$$
V(c)=\Phi^{-1}\left(D\left(\varphi(c), \frac{\varepsilon}{2}\right)\right) \cap\left(\varphi^{*}\right)^{-1}\left(D\left(\varphi(c), \frac{\varepsilon}{2}\right)\right) \cap U_{0}
$$

и определим $U$ как окрестность (в $X)$ множества $C$ такую, что $C \subset U \subset \mathrm{Cl}(U) \subset \cup\{V(c) \mid$ $\left.c \in \mathrm{Cl}_{A}(C)\right\} \subset U_{0}$. Тогда на паракомпакте $\mathrm{Cl}(U) \cup A$ отображение $\psi_{0}$, являющееся ограничением отображения $\varphi^{*}$, есть $\varepsilon$-селекция ограничения $\Phi$ на $\mathrm{Cl}(U) \cup A$. Действительно, для точек $x \in \mathrm{Cl}_{A}(U) \varphi^{*}(x)=\varphi(x) \in \Phi(x)$, а для точек $x \in \mathrm{Cl}(U)$ $\varphi^{*}(x)$ по построению лежит в некотором шаре радиуса $\varepsilon / 2$, которьй пересекается с множеством $\Phi(x)$. Построим последовательность $\psi_{0}, \psi_{1}, \ldots, \psi_{n}, \ldots$ (аналогично тому, как строилась последовательность $f_{0}, f_{1}, \ldots, f_{n}, \ldots$ в доказательстве случая 1$)$ теоремы 1 ) $\left(\lambda^{n} \varepsilon\right)$-селекций ограничения отображения $\Phi$ на паракомпакт $\mathrm{Cl}(U) \cup A$. При этом внесем дополнение, гарантирующее совпадение $\psi_{n}$ с $\varphi$ на множестве $A$. Для этого в индуктивном процессе определим многозначное отображение $\Phi_{n+1}: \mathrm{Cl}(U) \cup A \rightarrow Y$ равенством $\Phi_{n+1}(x)=\mathrm{Cl}\left(\left[D\left(\psi_{n}(x), \lambda^{n} \varepsilon\right) \cap \Phi(x)\right]\right)$. Тогда по индуктивному предположению $\varphi$ есть селекция $\left.\Phi_{n+1}\right|_{A}$. Затем определим отображение $\psi_{n+1}: \operatorname{Cl}(U) \cup A \rightarrow Y$ как непрерьвную селекцию полунепрерывного снизу многозначного отображения $\Psi_{n+1}$, совпадающего с $\varphi$ на $A$ и с $\Phi_{n+1}$ на $\mathrm{Cl}(U) \backslash A$. Тогда последовательность $\psi_{0}, \psi_{1}, \ldots, \psi_{n}, \ldots$ равномерно сходится к некоторой селекции $\psi$ ограничения отображения $\Phi$ на паракомпакт $\mathrm{Cl}(U) \cup A$. Тем самьм, свойство а) очевидно, а свойство в) следует из того, что 
$\mathrm{Cl}(U) \subset U_{0}$ и множество $U_{0}$ не пересекается с множеством $U_{-1} \supset A \backslash B$. Для доказательства свойства б) оценим расстояние $\|\psi(x)-\varphi(c)\|$ для $x \in V(c)$ и $c \in \mathrm{Cl}_{A}(C)$ :

$$
\begin{aligned}
\|\psi(x)-\varphi(c)\| & \leqslant\left\|\psi(x)-\varphi^{*}(x)\right\|+\left\|\varphi^{*}(x)-\varphi(c)\right\|<\sum_{n=0}^{\infty}\left\|\psi_{n+1}(x)-\psi_{n}(x)\right\|+\frac{\varepsilon}{2} \\
& \leqslant \frac{\varepsilon}{2}+\varepsilon\left(1+\lambda+\lambda^{2}+\cdots\right)=\varepsilon \frac{3-\lambda}{2(1-\lambda)}=R .
\end{aligned}
$$

Значит, шар $D(\psi(x), R)$ лежит в шаре $D(\varphi(c), 2 R)$, который по условию $\mu$-пересекает семейство $\{\Phi(x)\}, x \in X$. Следовательно, и шар $D(\psi(x), R) \quad \mu$-пересекает семейство $\{\Phi(x)\}, x \in X$. Предложение 1 доказано.

ДОКАЗАТЕЛЬСТВО ТЕОРЕМЫ 2. По аналогии с определением 2 семейство $\{L\}$ непустых замкнутых подмножеств банахова пространства назовем равностепенно локально паравыпуклым относительно подмножества $M \subset \cup\{L\}$, если для любой точки $y \in M$ найдутся $r=r(y)>0$ и $\alpha=\alpha(y) \in[0,1)$ такие, что шар $D(y, r)$ радиуса $r$ с центром в точке $y \quad \alpha$-пересекает семейство $\{L\}$. Нетрудно видеть, что в доказательстве теоремы 1 равностепенную локальную паравыпуклость всего семейства $\{L\}$ можно ослабить до условия равностепенной локальной паравьпуклости семейства $\{L\}$ относительно множества $h(A) \subset \cup\{L\}$ значений частичной селекции $h: A \rightarrow Y$. В свою очередь, неравенство

$$
\alpha_{\text {loc }}(y,\{L\})=\varlimsup_{r \rightarrow 0} \inf \{\alpha \geqslant 0\}<1,
$$

где инфимум берется по всем шарам $D(y, r), \alpha$-пересекающим $\{L\}$, означает наличие чисел $r=r(y)>0$ и $\alpha=\alpha(y) \in[0,1)$ таких, что шар $D(y, r)$ радиуса $r$ с центром в точке $y$ $\alpha$-пересекает семейство $\{L\}$. Другими словами, условие $\alpha_{\text {loc }}(h(a),\{L\})<1$ для всех $a \in A$ как раз и означает равностепенную локальную паравьпуклость семейства $\{L\}$ относительно множества $h(a) \subset \cup\{L\}$. Теорема 2 доказана.

4. В примерах ограничимся пространствами $Y$, норма которых задана скалярным произведением. Фиксируем разложение $Y=Z \oplus Z^{\perp}$ в прямую ортогональную сумму подпространств с $\operatorname{dim} Z^{\perp}=1$. Пусть $V \subset Z$ - замкнутое вьпуклое подмножество и $f: V \rightarrow Z^{\perp}-$ функция с фиксированной константой Липшица $k \geqslant 0$.

Лемма 2. Существует константа $\alpha(k) \in[0,1)$ такая, что график $P=\Gamma_{f}$ любой функции $f$ определенного выше типа есть $\alpha(k)$-паравыпуклое подмножсество $Y$ (независимо от $Z, V, f)$.

ДокАЗАТЕЛЬСТво. Пусть $x_{1}, x_{2}, \ldots, x_{n}$ - точки из $V$, а $y_{i}=\left(x_{i}, f\left(x_{i}\right)\right)$ - соответствуюшие точки на графике $\Gamma_{f}$. Рассмотрим (единственньй) чебьшевский центр $c$ многогранника $\left[y_{1}, y_{2}, \ldots, y_{n}\right]$. Можно считать, что $c$ лежит внутри $\left[y_{1}, y_{2}, \ldots, y_{n}\right]$ (иначе возможен переход к меньшей размерности), поэтому все расстояния $c y_{i}$ равны чебышевскому радиусу $R=R\left[y_{1}, y_{2}, \ldots, y_{n}\right]$ этого многогранника. Проведем через $c$ гиперплоскость П параллельную $Z$ и обозначим через $c^{*}$ соответствующую точку графика. Если $c \in \Pi$, то $c=c^{*} \in \Gamma_{f}$ и, тем самым, $\operatorname{dist}\left(c, \Gamma_{f}\right)=0$. Если же $c \notin \Pi$, то одна из точек, скажем $y_{1}$, лежит с точкой $c^{*}$ по разные стороны от П. Проведем двумерную плоскость $\Pi_{0}$ через точки $c, c^{*}, y_{1}$ и рассмотрим ограничение функции $f$ на пересечение $V \cap \Pi_{0}$. В плоскости $\Pi_{0}$ проведем угол величины $2 \operatorname{arctg} k$ с вершиной в точке $y_{1}$ и с биссектрисой, параллельной гиперплоскости П и пересекающей прямую, проходящую через точки $c$ 
и $c^{*}$. Тогда в силу липшицевости с константой $k$ функции $f$ обе точки $c$ и $c^{*}$ лежат в одной из половин этого угла. Поэтому $\Gamma_{f}$ пересекает перпендикуляр, опущенный из точки $c$ на "наклонную", т.е. непараллельную гиперплоскости П сторону этой половины. Осталось заметить, что длина этого перпендикуляра не больше $R \sin (\operatorname{arctg} k)$. Значит, $\operatorname{dist}\left(c, \Gamma_{f}\right) \leqslant R \sin (\operatorname{arctg} k)$. Для точки $y$ многогранника $\left[y_{1}, y_{2}, \ldots, y_{n}\right]$, отличной от $c$, возможны два случая: либо $y$ близка к $c$ и тогда оценка для $\operatorname{dist}\left(y, \Gamma_{f}\right)$ чуть хуже оценки для $\operatorname{dist}\left(c, \Gamma_{f}\right)$, либо расстояние $c y_{i}$ больше некоторой константы и тогда одно из расстояний $y y_{i}$ заметно меньше $R$ (подробнее см. [6, леммы 1,2$]$ ).

СлЕДСТВИЕ 1. Семейство всех графиков липиицевых с константой $k$ функиий с выпуклыми замкнутыми областями определения является равностепенно локально паравыпуклым (система координат не фиксирована).

Достаточно любопытным является следующее наблюдение: если в лемме $2 \operatorname{dim} Y=2$ и $f$ непрерьвно дифференцируемьм образом зависит от одной вещественной переменной $x$, то константа Липшица $k$ есть максимум модуля производной $f^{\prime}$ на соответствующем промежутке. Если фиксировать точку $x$ и рассмотреть оценку невьпуклости графика $\Gamma_{f}$ в малых окрестностях точки $x$, то получится величина типа $\sin \left(\operatorname{arctg} f^{\prime}(x)\right)$. При этом дифференцирование по $x$ последнего выражения дает в точности кривизну графика $\Gamma_{f}$ в точке $x$. Впрочем, за счет изменения системы координат локальная невыпуклость для графиков функций оценивается куда более радикальным образом.

Лемма 3. Локальная невыпуклость графика функиии $f: V \rightarrow Z^{\perp}, V \subset Z, Y=$ $Z \oplus Z^{\perp}$, равна 0 в любой точке графика, где сущ,ствует касательная.

ДокАЗАТЕЛЬСТво. В достаточно малых окрестностях такой точки расстояния от точек графика до касательной гиперплоскости есть величины, бесконечно малые по сравнению с радиусами окрестностей.

Пусть $Y$ - двумерное евклидово пространство и $f: Y \rightarrow \mathbb{R}$ - непрерывная функция. Назовем бескоординатным дифференииальным уравнением выражение $y^{\prime}(p)=f(p)$, $p \in Y$. Решением такого уравнения назовем пару $(T, \varphi)$, где $T: Y \rightarrow \mathbb{R}^{2}$ - изометрия евклидовых пространств и $\varphi: I \rightarrow \mathbb{R}$ - решение дифференциального уравнения $y^{\prime}(x)=f\left(T^{-1}(x, y(x))\right)$ для некоторого невырожденного замкнутого числового промежутка $I \subset \mathbb{R}, x \in I$. Графиком решения $(T, \varphi)$ бескоординатного дифференциального уравнения $y^{\prime}(p)=f(p), p \in Y$, назовем подмножество $T^{-1}\left(\Gamma_{\varphi}\right)$ пространства $Y$. Другими словами, мы рассматриваем связные замкнутые подмножества интегральных кривых некоторого фиксированного дифференциального уравнения первого порядка, разрешенного относительно производной, и к этим множествам применяем все возможные изометрии плоскости. Теорема Пеано гарантирует наличие (но не единственность) интегральных кривых, проходящих через фиксированную точку $(x, y) \in \mathbb{R}^{2}$.

СлЕДСТВИЕ 2. Пусть $f_{0}: Y \rightarrow[0, \infty)$ - локально ограниченная функиия $и C\left(Y, \leqslant f_{0}\right)$ - множество всех непрерывных функиий $f: Y \rightarrow \mathbb{R}$, которые по модулю мажсорируются функиией $f_{0}$. Тогда семейство графиков всех решений бескоординатных дифференииальных уравнений $y^{\prime}(p)=f(p), p \in Y$, с непрерывными правыми частями $f \in C\left(Y, \leqslant f_{0}\right)$ есть равностепенно локально паравыпуклое семейство подмножеств двумерного евклидова пространства $Y$.

ДокАЗАТЕЛЬСтво. Для фиксированной точки $p_{0} \in Y$ выберем окрестность $W$, в которой значения функции $f_{0}: Y \rightarrow \mathbb{R}$ по модулю не превосходят константы $k=\left|f\left(p_{0}\right)\right|+1$. 
Тогда для любой $f \in C\left(Y, \leqslant f_{0}\right)$ и любого решения $(T, \varphi)$ уравнения $y^{\prime}(p)=f(p)$, график которого $T^{-1}\left(\Gamma_{\varphi}\right)$ проходит через точку $p_{0}$, пересечение этого графика с окрестностью $W$ есть (в подходящей системе координат) график липшицевой с константой $k$ функции с вьпуклой замкнутой областью определения. По лемме 2 все такие пересечения есть $\alpha(k)$-паравыпуклые подмножества $Y$ для некоторой константы $\alpha(k)<1$.

Верен и аналог следствия 2 для $(n+1)$-мерного евклидова пространства $Y$. Следует только заменить функции $f: Y \rightarrow \mathbb{R}$ отображениями $f$ из $Y$ в пространство $\mathbb{R}^{n}$, интерпретируемое как пространство $L\left(\mathbb{R}^{n}, \mathbb{R}\right)$ всех линейных отображений из $\mathbb{R}^{n}$ в $\mathbb{R}$. При этом решением такого бескоординатного дифференциального уравнения является пара $(T, \varphi)$, где $T: Y \rightarrow \mathbb{R}^{n}$ - изометрия евклидовых пространств и $\varphi: \Delta \rightarrow \mathbb{R}$ - решение дифференциального уравнения $y^{\prime}(x)=f\left(T^{-1}(x, y(x))\right)$ для некоторого вьпуклого замкнутого тела $\Delta \subset \mathbb{R}^{n}, x \in \Delta$. Селекционная теорема 1 в случае гильбертова пространства говорит о возможности непрерывного (локального) выбора начальных условий в зависимости от решения: на каждой интегральной кривой непрерывным образом выбирается по точке.

В заключение сформулируем открытьй вопрос.

Вопрос. Является ли паравыпуклымграфик лишшицева с константой $f$ отображения (не функции) $f: \mathbb{R}^{n} \rightarrow \mathbb{R}^{m}$ ?

Ответ неизвестен даже при $n=1$ и $m=2$. Формальное препятствие состоит в неприменимости техники работы [7]. Отметим также, что в определениях 1,2 и теоремах 1,2 возможна замена паравыпуклости с некоторой константой из $[0,1)$ на функииональную паравьпуклость в стиле работ [8]-[10].

\section{СПИСОК ЦИТИРОВАННОЙ ЛИТЕРАТУРЫ}

[1] Michael E. Continuous selections. II // Ann. of Math. 1956. V. 64. P. 562-580.

[2] Pixley C. An example concerning continuous selections on infinite dimensional spaces // Proc. Amer. Math. Soc. 1974. V. 43. P. 237-244.

[3] Michael E. Selection theorems with and without dimensional restrictions // Internat. Conference in Memory of F. Hausdorff. Math. Res. V. 67. Berlin: Akademie-Verlag, 1992. P. 218-222.

[4] Michael E. Continuous selections. I // Ann. of Math. 1956. V. 63. P. 361-382.

[5] Michael E. Paraconvex sets // Math. Scand. 1959. V. 7. P. 372-376.

[6] Семёнов П. В. О паравьпуклости звездноподобных множеств // Сиб. матем. ж. 1996. Т. 37. № 2. C. 399-405.

[7] Семёнов П. В. Выпуклые сечения граффиков непрерьвных функций // Матем. заметки. 1991. T. 50. № 5. C. 75-80.

[8] Repovš D., Semenov P. V. On paraconvexity of graphs of continuous functions // Set-Valued Anal. 1995. V. 3. P. 23-32.

[9] Repovš D., Semenov P. V. On functions of nonconvexity for graphs of continuous functions // J. Math. Anal. Appl. 1995. V. 196. P. 1021-1029.

[10] Семёнов П. В. Функционально паравыпуклые множества // Матем. заметки. 1993. Т. 54. № 6. C. $74-91$. 